\title{
Symmetries, conservation laws, and 'integrability' of difference equations
}

\author{
Mensah K Folly-Gbetoula' and Abdul H Kara ${ }^{1,2^{*}}$
}

\section{"Correspondence:}

Abdul.Kara@wits.ac.za

${ }^{1}$ School of Mathematics, University of the Witwatersrand, 1 Jan Smuts, Johannesburg, South Africa

${ }^{2}$ Department of Mathematics and

Statistics, University of Petroleum

and Minerals, Dhahran, Saudi Arabia

\begin{abstract}
A number of nontrivial conservation laws of some difference equations, viz., the discrete Liouville equation and the discrete sine-Gordon equation, are constructed using first principles. Symmetries and the more recent ideas and notions of characteristics (multipliers) for difference equations are also discussed.
\end{abstract}

Keywords: conservation laws; symmetries; difference equations; discrete Liouville equation; discrete sine-Gordon equation

\section{Introduction}

The role of symmetries of difference equations is now well established and the applications of the symmetries in the analysis (especially reduction) of the equations are also well documented (see [1-4]). However, the role and construction of conservation laws for partial difference equations (PDEs), to the best of our knowledge, is somewhat new but the preliminary concepts and definitions are available even in the context of variational equations (see [5-7]). These conservation laws, as in the case of differential equations, have a variety of applications especially as another tool in the reduction of the equation under scrutiny.

The aim of this work is to obtain the conservation laws of PDEs which are of interest, viz., the discrete Liouville equation and the discrete sine-Gordon equation. These equations were studied in $[8]$ and $[9,10]$, inter alia. The method for the construction of the conservation laws employed here follows that introduced in [5]. The variational approach, not followed here, uses the equivalent of Noether symmetries and can be found in [7].

We regard the domain of a given partial differential equation (PDE) as a fiber bundle $M=X \times U$, where $X$ is the base space of independent variables and $U$ is the vertical space, i.e., the fiber of dependent variables $\mathbf{u}$ over each $\mathbf{x} \in X$. The direct method for constructing conservation laws of PDEs requires the domain $M$ to be topologically trivial, which occurs if each fiber $U$ and the base space $X$ are star-shaped (see Poincarés lemma).

Symmetries and conservation laws are useful tools for finding exact solutions to differential equations. The association of symmetries, conservation laws and integrability was established for differential equations [11]. It has been shown that when the symmetry generator and the first integral (conservation laws) are associated via the invariance condition, one may proceed to double reduction of the equation. Consequently, these properties should be retained when discrete analogs of such equations are constructed. Several 
methods for obtaining a discretization of a differential equation have been studied (see [12-15]).

As far as PDEs are concerned, we can write the domain as $M=X \times U$, but now $X$ is the set of integer-valued multi-indices $\mathbf{n}$ that label each lattice point (we assume that the lattice points are labeled sequentially, without jumps; this does not require the lattice to be uniform).

\section{Conservation laws of the discrete Liouville equation and the discrete sine-Gordon equation}

Let us consider the difference equation

$$
u_{k+1}^{l+1}=\omega\left(k, l, u_{k}^{l}, u_{k}^{l+1}, u_{k+1}^{l}\right) \text {, }
$$

where $k$ and $l$ are integers, $u_{k}^{l}$ is a function that depends on the independent variables $k$ and $l, \omega$ is a function of the dependent and independent variables. Finding the conservation laws requires the knowledge of the shift operators. They are defined as follows:

$$
S_{m}: u_{m}^{n} \mapsto u_{m+1}^{n}, \quad S_{n}: u_{m}^{n} \mapsto u_{m}^{n+1} .
$$

A conservation law for the PDE is an expression of the form

$$
\left(S_{k}-\mathrm{id}\right) F+\left(S_{l}-\mathrm{id}\right) G=0
$$

that is satisfied by all solutions of the equation [5]. Note that $F$ and $G$ are functions of the dependent and independent variables, id is the identity mapping. We are looking for conservation laws that lie on the quad-graph and we are interested in finding nontrivial conservation laws. We then assume that the functions $F$ and $G$ are of the form

$$
F=F\left(k, l, u_{k}^{l}, u_{k}^{l+1}\right), \quad G=G\left(k, l, u_{k}^{l}, u_{k+1}^{l}\right) .
$$

The conservation laws (3) amount to

$$
\left(S_{k}-\mathrm{id}\right) F\left(k, l, u_{k}^{l}, u_{k}^{l+1}\right)+\left(S_{l}-\mathrm{id}\right) G\left(k, l, u_{k}^{l}, u_{k+1}^{l}\right)=0 .
$$

In order to find $F$ and $G$, we consider the theory provided by Hydon in [5]. In his paper, Hydon applied the method to scalar partial difference equations that are second order in one variable but in this paper we are dealing with first order difference equations in two variables.

\subsection{The discrete Liouville equation}

Consider the discrete Liouville equation

$$
u_{k+1}^{l+1}=\frac{u_{k}^{l+1} u_{k+1}^{l}+z_{k}^{l}}{u_{k}^{l}}
$$

It is an equation which is not integrable through the inverse scattering transform techniques (see [8]). The application of this method to (6) requires $z_{k}^{l}$ to satisfy

$$
z_{k+1}^{l+1} z_{k}^{l}-z_{k}^{l+1} z_{k+1}^{l}=0 .
$$


We therefore assume that condition (7) holds for any $k$ and $l$. Note that (3) can be written as follows:

$$
F\left(k+1, l, u_{k+1}^{l}, \omega\right)-F\left(k, l, u_{k}^{l}, u_{k}^{l+1}\right)+G\left(k, l+1, u_{k}^{l+1}, \omega\right)-G=0,
$$

where $\omega$ is the right-hand side of the discrete Liouville (6).

As we said earlier, we are not interested in nontrivial conversation laws. It is worthwhile to mention that if $F$ and $G$ are solutions to (8), then $F+F_{1}(k, l)$ and $G+G_{1}(k, l)$ are also solutions for any functions $F_{1}$ and $G_{1}$. One can see from (8) that $F$ and $G$ take different arguments. To overcome this, we eliminate terms that depend on $\omega$, by differentiating with respect to $u_{k}^{l+1}$ and $u_{k+1}^{l}$, respectively, keeping omega fixed. The derivative of (8) with respect to $u_{k+1}^{l}$ is given by

$$
\frac{u_{k}^{l} u_{k}^{l+1}}{u_{k}^{l+1} u_{k+1}^{l}+z_{k}^{l}}\left[-F_{, 3}-G_{, 3}\right]+F_{, 3}\left(k+1, l, u_{k+1}^{l}, \omega\right)-G_{, 4}=0 .
$$

The differentiation of (8) with respect to $u_{k}^{l+1}$ leads to

$$
\frac{u_{k}^{l} u_{k+1}^{l}}{u_{k}^{l+1} u_{k+1}^{l}+z_{k}^{l}}\left[-F_{, 3}-G_{, 3}\right]-F_{, 4}+G_{, 3}\left(k, l+1, u_{k}^{l+1}, \omega\right)=0 .
$$

Differentiating (10) with respect to $u_{k+1}^{l}$ we get

$$
\begin{aligned}
& \frac{u_{k}^{l} u_{k}^{l+1}}{u_{k}^{l+1} u_{k+1}^{l}+z_{k}^{l}}\left[\frac{-u_{k+1}^{l}\left(F_{, 3}+G_{, 3}\right)}{u_{k}^{l+1} u_{k+1}^{l}+z_{k}^{l}}-\frac{u_{k}^{l} u_{k+1}^{l}\left(F_{, 33}+G_{, 33}\right)}{u_{k}^{l+1} u_{k+1}^{l}+z_{k}^{l}}-F_{, 43}\right] \\
& \quad+\frac{z_{k}^{l} u_{k}^{l}\left(-F_{, 3}-G_{3}\right)}{\left(u_{k}^{l+1} u_{k+1}^{l}+z_{k}^{l}\right)^{2}}-\frac{u_{k}^{l} u_{k+1}^{l} G_{34}}{u_{k}^{l+1} u_{k+1}^{l}+z_{k}^{l}}=0 .
\end{aligned}
$$

We multiply by $\left(u_{k}^{l+1} u_{k+1}^{l}+z_{k}^{l}\right)^{2}$ to clear fractions. This gives

$$
\begin{aligned}
& \left(u_{k}^{l+1} u_{k+1}^{l}+z_{k}^{l}\right)\left(F_{, 3}+G_{, 3}\right)+u_{k}^{l} u_{k+1}^{l} u_{k}^{l+1}\left(F_{, 33}+G_{, 33}\right) \\
& \quad+u_{k}^{l+1}\left(u_{k}^{l+1} u_{k+1}^{l}+z_{k}^{l}\right) F_{, 43}+u_{k+1}^{l}\left(u_{k}^{l+1} u_{k+1}^{l}+z_{k}^{l}\right) G_{43}=0 .
\end{aligned}
$$

In order to get rid of $G$ we differentiate two times with respect to $u_{k}^{l+1}$. This yields

$$
\begin{aligned}
& u_{k+1}^{l}\left(F_{, 3}+G_{, 3}\right)+u_{k}^{l} u_{k+1}^{l}\left(F_{, 33}+G_{, 33}\right)+u_{k}^{l} u_{k}^{l+1} u_{k+1}^{l} F_{, 334} \\
& \quad+\left(3 u_{k}^{l+1} u_{k+1}^{l}+2 z_{k}^{l}\right) F_{, 43}+u_{k}^{l+1}\left(u_{k}^{l+1} u_{k+1}^{l}+z_{k}^{l}\right) F_{, 344}+u_{k+1}^{l}{ }^{2} G_{, 34}=0
\end{aligned}
$$

and

$$
\begin{aligned}
& 4 u_{k+1}^{l} F_{, 34}+2 u_{k}^{l} u_{k+1}^{l} F_{, 334}+\left(5 u_{k}^{l+1} u_{k+1}^{l}+3 z_{k}^{l}\right) F_{, 334}+u_{k}^{l} u_{k}^{l+1} u_{k+1}^{l} F_{, 3344} \\
& \quad+\left(u_{k}^{l+1^{2}} u_{k+1}^{l}+z_{k}^{l} u_{k}^{l+1}\right) F_{, 3444}=0
\end{aligned}
$$

respectively. The function $F$ does not depend on $u_{k}^{l+1}$, therefore we can separate by powers of $u_{k}^{l+1}$ to get

$$
\text { 1: } \quad 3 F_{, 344}+u_{k}^{l+1} F_{, 3444}=0,
$$




$$
u_{k+1}^{l}: \quad 4 F_{, 34}+2 u_{k}^{l} F_{, 334}+2 u_{k}^{l+1} F_{, 344}+u_{k}^{l} u_{k}^{l+1} F_{, 3344}=0 .
$$

From (15) we get

$$
F\left(k, l, u_{k}^{l}, u_{k}^{l+1}\right)=\frac{f_{1}\left(k, l, u_{k}^{l}\right)}{2 u_{k}^{l+1}}+f_{2}\left(k, l, u_{k}^{l}\right) u_{k}^{l+1}+f_{3}\left(k, l, u_{k}^{l}\right)+f_{4}\left(k, l, u_{k}^{l+1}\right)
$$

where $f_{1}, f_{2}, f_{3}$ and $f_{4}$ are arbitrary functions. On the other hand, we differentiate (11) with respect to $u_{k+1}^{l}$ to get an equation involving $G$ only. The first derivative with respect to $u_{k+1}^{l}$ gives

$$
\begin{gathered}
-u_{k}^{l+1}\left(F_{, 3}+G_{, 3}\right)-\left(u_{k}^{l+1} u_{k+1}^{l}+z_{k}^{l}\right) G_{, 34}-u_{k}^{l} u_{k}^{l+1}\left(F_{, 33}+G_{, 33}\right)-u_{k}^{l} u_{k}^{l+1} u_{k+1}^{l} G_{, 334} \\
-\left(2 u_{k}^{l+1} u_{k+1}^{l}+z_{k}^{l}\right) G_{, 43}-u_{k+1}^{l}\left(u_{k}^{l+1} u_{k+1}^{l}+z_{k}^{l}\right) G_{, 344}-u_{k}^{l+1^{2}} F_{, 34}=0
\end{gathered}
$$

and the second derivative gives

$$
\begin{gathered}
-4 u_{k}^{l+1} G_{, 34}\left(k, l, u_{k}^{l}, u_{k+1}^{l}\right)-\left(5 u_{k}^{l+1} u_{k+1}^{l}+3 z_{k}^{l}\right) G_{, 344}-2 u_{k}^{l+1} u_{k}^{l} G_{, 334} \\
-u_{k}^{l} u_{k}^{l+1} u_{k+1}^{l} G_{, 3344}-u_{k+1}^{l}\left(u_{k}^{l+1} u_{k+1}^{l}+z_{k}^{l}\right) G_{, 3444}=0 .
\end{gathered}
$$

Similarly, the function $G$ does not depend on $u_{k+1}^{l}$. This allows us to equate the coefficients of powers of $u_{l}^{k+1}$ to zero. We get

$$
\begin{aligned}
& 3 G_{, 344}+u_{k+1}^{l} G_{, 3444}=0, \\
& 4 G_{, 34}+5 u_{k+1}^{l} G_{, 344}+2 u_{k}^{l} G_{, 334}+u_{k}^{l} u_{k+1}^{l} G_{, 3344}+u_{k+1}^{l}{ }^{2} G_{, 3444}=0 .
\end{aligned}
$$

The general solution of (20) and (21) is given by

$$
G=\frac{g_{1}\left(k, l, u_{k}^{l}\right)}{2 u_{k+1}^{l}}+g_{2}\left(k, l, u_{k}^{l}\right) u_{k+1}^{l}+g_{3}\left(k, l, u_{k}^{l}\right)+g_{4}\left(k, l, u_{k+1}^{l}\right),
$$

where $g_{1}, g_{2}, g_{3}$, and $g_{4}$ are arbitrary functions. Substituting the expressions of $F$ and $G$, given by (17) and (22), into (14) and (19) gives

$$
f_{2}\left(k, l, u_{k}^{l}\right)=-\frac{c_{1}(k, l)}{u_{k}^{l}}+c_{2}(k, l)
$$

and

$$
g_{2}\left(k, l, u_{k}^{l}\right)=-\frac{c_{3}(k, l)}{u_{k}^{l}}+c_{4}(k, l)
$$

respectively. Therefore, we have

$$
\begin{aligned}
& F\left(k, l, u_{k}^{l}, u_{k}^{l+1}\right)=\frac{f_{1}}{2 u_{k}^{l+1}}+\left(-\frac{c_{1}(k, l)}{u_{k}^{l}}+c_{2}(k, l)\right) u_{k}^{l+1}+f_{3}+f_{4}, \\
& G\left(k, l, u_{k}^{l}, u_{k+1}^{l}\right)=\frac{g_{1}}{2 u_{k+1}^{l}}+\left(-\frac{c_{3}(k, l)}{u_{k}^{l}}+c_{4}(k, l)\right) u_{k+1}^{l}+g_{3}+g_{4} .
\end{aligned}
$$


Substituting the above results into (18), and separating by powers of $u_{k+1}^{l}$ and $u_{k}^{l+1}$, we obtain

$$
\begin{aligned}
& \text { 1: } \frac{f_{1}^{\prime \prime}}{2} u_{k}^{l}+2 c_{3} \frac{z_{k}^{l}}{u_{k}^{l^{2}}}=0, \\
& u_{k+1}^{l} u_{k}^{l+1}: \quad \frac{c_{3}}{u_{k}^{l^{2}}}-\frac{c_{3}}{u_{k}^{l^{2}}}=0, \\
& u_{k}^{l+1}: \quad f_{3}^{\prime}+g_{3}^{\prime}+u_{k}^{k}\left(f_{3}^{\prime \prime}+g_{3}^{\prime \prime}\right)=0, \\
& u_{k}^{l+1^{2}}: \quad \frac{c_{1}}{u_{k}^{l^{2}}}-\frac{c_{1}}{u_{k}^{l^{2}}}=0,
\end{aligned}
$$

and therefore

$$
f_{1}\left(k, l, u_{k}^{l}\right)=-\frac{2 c_{3} z_{k}^{l}}{u_{k}^{l}}+c_{5} u_{k}^{l}+c_{6}, \quad\left(f_{3}+g_{3}\right)\left(k, l, u_{k}^{l}\right)=c_{7} \ln \left(u_{k}^{l}\right)+c_{8} .
$$

On the other hand, substituting (23) and (24) into (13), and separating by powers of $u_{k}^{l+1}$, $u_{k+1}^{l}$, we get (after solving the resulting system)

$$
g_{1}\left(k, l, u_{k}^{l}\right)=-\frac{2 c_{1} z_{k}^{l}}{u_{k}^{l}}+c_{9} u_{k}^{l}+c_{10} .
$$

Results (23), (24), (25), and (26) lead to

$$
\begin{aligned}
F\left(k, l, u_{k}^{l}, u_{k}^{l+1}\right)= & \left(-\frac{2 c_{3}(k, l) z_{k}^{l}}{u_{k}^{l}}+c_{5}(k, l) u_{k}^{l}+c_{6}(k, l)\right) \frac{1}{2 u_{k}^{l+1}} \\
& -\left(\frac{c_{1}}{u_{k}^{l}}-c_{2}\right) u_{k}^{l+1}+f_{3}\left(k, l, u_{k}^{l}\right)+f_{4}\left(k, l, u_{k}^{l+1}\right)
\end{aligned}
$$

and

$$
\begin{aligned}
G\left(k, l, u_{k}^{l}, u_{k+1}^{l}\right)= & \left(-\frac{2 c_{1} z_{k}^{l}}{u_{k}^{l}}+c_{9} u_{k}^{l}+c_{10}\right) \frac{1}{2 u_{k+1}^{l}}+\left(-\frac{c_{3}}{u_{k}^{l}}+c_{4}\right) u_{k+1}^{l} \\
& -f_{3}\left(k, l, u_{k}^{l}\right)+c_{7} \ln \left(u_{k}^{l}\right)+c_{8}+g_{4}\left(k, l, u_{k+1}^{l}\right),
\end{aligned}
$$

where $c_{i}, 1 \leq i \leq 10$, depend on $k$ and $l$. The substitution of (27) and (28) in (11) did not provide any new information on the unknown functions. We then continue our computations by substituting (27) and (28) into (9), and separating the resulting equation by powers of $u_{k}^{l+1}$. This yields

$$
\begin{gathered}
1: \quad z_{k}^{l} f_{3}^{\prime}\left(k+1, l, u_{k+1}^{l}\right)-c_{4} z_{k}^{l}-z_{k}^{l} g_{4}^{\prime}\left(k, l, u_{k+1}^{l}\right)+\frac{c_{10} z_{k}^{l}}{2 u_{k+1}^{l} 2}+\frac{z_{k}^{2^{2}} c_{1}(k+1, l)}{u_{k}^{l} u_{k+1}^{l}{ }^{2}} \\
-\frac{z_{k}^{l^{2} c_{1}}}{u_{k}^{l} u_{k+1}^{l}{ }^{2}}+\frac{\left(-c_{5}+c_{5}(k+1, l)\right) u_{k}^{l}}{2}+\frac{\left(2 c_{3}(k+1, l) z_{k+1}^{l}+c_{9} z_{k}^{l}\right) u_{k}^{l}}{2 u_{k+1}^{l}}=0, \\
u_{k}^{l+1}: \quad-c_{7}+u_{k+1}^{l} f_{3}^{\prime}\left(k+1, l, u_{k+1}^{l}\right)-c_{4} u_{k+1}^{l}-u_{k+1}^{l} g_{4}^{\prime}\left(k, l, u_{k+1}^{l}\right)+\frac{c_{10}}{2 u_{k+1}^{l}} \\
\quad-\frac{2 c_{9} u_{k}^{l}}{2 u_{k+1}^{l}}+\frac{c_{9} u_{k}^{l}}{2 u_{k+1}^{l}}-\frac{c_{1} z_{k}^{l}}{u_{k}^{l} u_{k+1}^{l}}+\frac{2 c_{1}(k+1, l) z_{k}^{l}}{u_{k}^{l} u_{k+1}^{l}}-\frac{c_{1} z_{k}^{l}}{u_{k}^{l} u_{k+1}^{l}}=0,
\end{gathered}
$$


where ' denotes the derivative with respect to the dependent variable. Again we can split (29) and (30) by powers of $u_{k}^{l}$ since $f_{3}, g_{4}$, and $c_{i}, 1 \leq i \leq 10$, do not depend on $u_{k}^{l}$. We redo the same thing with (10) to get

$$
\begin{aligned}
& 1: \quad- z_{k}^{l} f_{4}^{\prime}\left(k, l, u_{k}^{l+1}\right)-c_{2} z_{k}^{l}+z_{k}^{l} g_{3}^{\prime}\left(k, l+1, u_{k}^{l+1}\right)+\frac{c_{6} z_{k}^{l}}{2 u_{k}^{l+1^{2}}} \frac{z_{k}^{l^{2}} c_{3}(k, l+1)}{u_{k}^{l} u_{k}^{l+1^{2}}} \\
&- \frac{z_{k}^{l^{2}} c_{3}}{u_{k}^{l} u_{k}^{l+1^{2}}}+\frac{\left(-c_{9}(k, l)+c_{9}(k, l+1)\right) u_{k}^{l}}{2}+\frac{\left(2 c_{1}(k, l+1) z_{k}^{l+1}+c_{5} z_{k}^{l}\right) u_{k}^{l}}{2 u_{k}^{l+1^{2}}}=0, \\
& u_{k+1}^{l}: \quad-c_{7}+u_{k+1}^{l} f_{3}^{\prime}\left(k+1, l, u_{k+1}^{l}\right)-c_{4} u_{k+1}^{l}-u_{k+1}^{l} g_{4}^{\prime}\left(k, l, u_{k+1}^{l}\right) \\
& \quad+\frac{c_{10}}{2 u_{k+1}^{l}}-\frac{2 c_{9} u_{k}^{l}}{2 u_{k+1}^{l}}+\frac{c_{9} u_{k}^{l}}{2 u_{k+1}^{l}}-\frac{c_{1} z_{k}^{l}}{u_{k}^{l} u_{k+1}^{l}}+\frac{2 c_{1}(k+1, l) z_{k}^{l}}{u_{k}^{l} u_{k+1}^{l}}-\frac{c_{1} z_{k}^{l}}{u_{k}^{l} u_{k+1}^{l}}=0 .
\end{aligned}
$$

After rearranging, simplifying, and solving the resulting (29), (30), (31), and (32), we get some new information on the unknown functions:

$$
\begin{aligned}
& c_{1}(k, l)=K_{1}, \quad c_{3}(k, l)=K_{3}, \quad c_{5}(k, l)=-\frac{2 c_{1}(k, l+1) k_{k}^{l+1}}{z_{k}^{l}}, \quad c_{7}=0, \\
& c_{9}(k, l)=-2 \frac{c_{3}(k+1, l) z_{k+1}^{l}}{z_{k}^{l}}, \quad g_{3}\left(k, l+1, u_{k}^{l+1}\right)=f_{4}\left(k, l, u_{k}^{l+1}\right)+\frac{c_{6}}{2 u_{k}^{l+1}}+c_{2} u_{k}^{l+1}+c_{12}, \\
& f_{3}\left(k+1, l, u_{k+1}^{l}\right)=g_{4}\left(k, l, u_{k+1}^{l}\right)+\frac{c_{10}}{2 u_{k+1}^{l}}+c_{4} u_{k+1}^{l}+c_{11} .
\end{aligned}
$$

The last step consists of substituting all the information we got so far into (8). This yields

$$
\begin{aligned}
& c_{5}(k, l)=K_{5}, \quad c_{3}(k, l)=K_{3}, \quad c_{1}(k, l)=K_{1}, \\
& c_{9}(k, l)=K_{9}, \quad c_{13}(k, l)=0, \quad c_{4}+c_{14}=-K_{2}, \\
& c_{11}+c_{15}(k+1, l)-c_{16}(k-1, l)-c_{11}(k-1, l)+c_{12}+c_{16}(k, l-1)-c_{12}(k, l-1)=0,
\end{aligned}
$$

where $K_{1}, K_{3}, K_{5}$, and $K_{9}$ are constants. Summarizing these results, we have obtained the conservation laws

$$
\begin{aligned}
F\left(k, l, u_{k}^{l}, u_{k}^{l+1}\right)= & -K_{1}\left(\frac{z_{k}^{l+1} u_{k}^{l}}{z_{k}^{l} u_{k}^{l+1}}+\frac{u_{k}^{l+1}}{u_{k}^{l}}\right)+K_{2} u_{k}^{l+1}-K_{2}(k-1, l) u_{k}^{l} \\
& -K_{3} \frac{z_{k}^{l}}{u_{k}^{l} u_{k}^{l+1}}+c_{16}(k-1, l)+c_{11}(k-1, l)+c_{15}, \\
G\left(k, l, u_{k}^{l}, u_{k+1}^{l}\right)= & -K_{1} \frac{z_{k}^{l}}{u_{k}^{l} u_{k+1}^{l}}-K_{3}\left(\frac{z_{k+1}^{l} u_{k}^{l}}{z_{k}^{l} u_{k+1}^{l}}+\frac{u_{k+1}^{l}}{u_{k}^{l}}\right)-K_{2} u_{k+1}^{l} \\
& -K_{2}(k, l-1) u_{k}^{l}-c_{15}(k, l-1)+c_{12}(k, l-1)+c_{16} .
\end{aligned}
$$

Therefore all independent conservations laws for the discrete Liouville equation are given by

$$
F_{1}\left(k, l, u_{k}^{l}, u_{k}^{l+1}\right)=-\frac{z_{k}^{l+1} u_{k}^{l}}{z_{k}^{l} u_{k}^{l+1}}-\frac{u_{k}^{l+1}}{u_{k}^{l}}, \quad G_{1}\left(k, l, u_{k}^{l}, u_{k+1}^{l}\right)=-\frac{z_{k}^{l}}{u_{k}^{l} u_{k+1}^{l}} ;
$$




$$
\begin{aligned}
& F_{2}=K_{2} u_{k}^{l+1}-K_{2}(k-1, l) u_{k}^{l}, \quad G_{2}=-K_{2} u_{k+1}^{l}-K_{2}(k, l-1) u_{k}^{l} ; \\
& F_{3}\left(k, l, u_{k}^{l}, u_{k}^{l+1}\right)=-\frac{z_{k}^{l}}{u_{k}^{l} u_{k}^{l+1}}, \quad G_{3}\left(k, l, u_{k}^{l}, u_{k+1}^{l}\right)=-\frac{z_{k+1}^{l} u_{k}^{l}}{z_{k}^{l} u_{k+1}^{l}}-\frac{u_{k+1}^{l}}{u_{k}^{l}},
\end{aligned}
$$

where $K_{2}$ is a function of $k$ and $l$. Note that $\left(F_{2}, G_{2}\right)$ is a trivial one.

\subsection{The discrete sine-Gordon equation}

The discrete sine-Gordon equation is given by $[9,10]$

$$
u_{k+1}^{l+1} u_{k}^{l}=\frac{1+z_{k}^{l} u_{k}^{l+1} u_{k+1}^{l}}{u_{k}^{l+1} u_{k+1}^{l}+z_{k}^{l}}
$$

Again, $z_{i}^{j}$ must satisfy

$$
z_{k}^{l} z_{k+1}^{l+1}=z_{k}^{l+1} z_{k+1}^{l}
$$

Equation (38) represents a nonautonomous extension of the lattice sine-Gordon equation. In the continuous limit, this nonautonomous form goes over to $w_{x, t}=f(x) g(t) \sin w$ (this explicit $x$ and $t$ dependence can be absorbed through a redefinition of the independent variables leading to the standard, autonomous, sine-Gordon case, but no such gauge exists in the discrete case [8]).

In this section, we jump the first few steps since we have explained them in the first example. Let us take it from the condition of the conservation laws (8),

$$
F\left(k+1, l, u_{k+1}^{l}, \omega\right)-F\left(k, l, u_{k}^{l}, u_{k}^{l+1}\right)+G\left(k, l+1, u_{k}^{l+1}, \omega\right)-G=0,
$$

where $\omega$ is given by

$$
\omega=u_{k+1}^{l+1}=\frac{1+z_{k}^{l} u_{k}^{l+1} u_{k+1}^{l}}{u_{k}^{l}\left(u_{k}^{l+1} u_{k+1}^{l}+z_{k}^{l}\right)} .
$$

We differentiate (40) with respect to $u_{k}^{l+1}$ by applying the operator $\left(\partial / \partial u_{k}^{l+1}\right)-\left(\omega_{u_{k}^{l+1}} / \omega_{u_{k}^{l}}\right) \times$ $\left(\partial / \partial u_{k}^{l}\right)$ to get

$$
\begin{aligned}
& \left(z_{k}^{l^{2}}-1\right) u_{k}^{l} u_{k+1}^{l}\left[F_{, 3}\left(k, l, u_{k}^{l}, u_{k}^{l+1}\right)+G_{, 3}\left(k, l, u_{k}^{l}, u_{k+1}^{l}\right)\right] \\
& \quad+\left(z_{k}^{l} u_{k}^{l+1} u_{k+1}^{l}{ }^{2}+u_{k}^{l+1} u_{k+1}^{l}+z_{k}^{l^{2}} u_{k}^{l+1} u_{k+1}^{l}+z_{k}^{l}\right)\left[F_{, 4}-G_{, 3}\left(k, l+1, u_{k}^{l+1}, \omega\right)\right]=0 .
\end{aligned}
$$

We repeat the same procedure but now we are planning to eliminate $F$ to obtain an equation that involves $G$ only. For the same reason as before, we equate coefficients of powers of $u_{k}^{l+1}$. The resulting system can be summarized as follows:

$$
\begin{aligned}
& 4 G_{, 3444}+u_{k+1}^{l} G_{, 34444}=0 \\
& \left(9 z_{k}^{l^{2}}+3\right) G_{344}+\left(7 z_{k}^{l^{2}}+3\right) u_{k+1}^{l} G_{, 3444}+\left(3 z_{k}^{l^{2}}-3\right) u_{k}^{l} G_{, 3344} \\
& \quad+\left(z_{k}^{l^{2}}-1\right) u_{k}^{l} u_{k+1}^{l} G_{, 33444}+\left(z_{k}^{l^{2}}+1\right) u_{k+1}^{l}{ }^{2} G_{, 34444}=0 \\
& 12 G_{, 344}+8 u_{k+1}^{l} G_{, 3444}+u_{k+1}^{l}{ }^{2} G_{, 34444}=0
\end{aligned}
$$


whose solution is given by

$$
G=\frac{g_{1}\left(k, l, u_{k}^{l}\right)}{u_{k+1}^{l}}+g_{2}\left(k, l, u_{k}^{l}\right) u_{k+1}^{l}+g_{3}\left(k, l, u_{k}^{l}\right)+g_{4}\left(k, l, u_{k+1}^{l}\right),
$$

for some functions $g_{1}, g_{2}, g_{3}$ and $g_{4}$. To know the dependency among the $g_{i}$ 's, $1 \leq i \leq 4$, we have to substitute $F$ and $G$ into the previous equations. After a set of long calculations, we find that the conservation laws for the discrete sine-Gordon equation are given by

$$
\begin{aligned}
& F_{1}=\frac{-c_{1}(k+1, l)}{\left(z_{k+1}^{l}-1\right) u_{k}^{l} u_{k}^{l+1}}-\frac{c_{1} u_{k}^{l} u_{k}^{l+1}}{z_{k}^{l^{2}}-1}, \quad G_{1}=\frac{-z_{k}^{l} c_{1} u_{k}^{l}}{\left(z_{k}^{l 2}-1\right) u_{k+1}^{l}}-\frac{c_{1}(k+1, l) z_{k}^{l} u_{k+1}^{l}}{\left(z_{k+1}^{l}{ }^{2}-1\right) u_{k}^{l}} ; \\
& F_{2}=u_{k}^{l+1}-u_{k}^{l}, \quad G_{2}=-u_{k+1}^{l}+u_{k}^{l} ; \\
& F_{3}=\frac{-z_{k}^{l} c_{3} u_{k}^{l}}{\left(z_{k}^{l^{2}}-1\right) u_{k}^{l+1}}+\frac{z_{k}^{l} c_{3}(k, l-1) u_{k}^{l+1}}{\left(1-z_{k}^{l-1}\right) u_{k}^{l}}, \quad G_{3}=\frac{c_{3}(k, l-1)}{\left(1-z_{k}^{l-1}\right) u_{k}^{l} u_{k+1}^{l}}-\frac{c_{3} u_{k}^{l} u_{k+1}^{l}}{\left(z_{k}^{l^{2}}-1\right)},
\end{aligned}
$$

where $c_{1}$ and $c_{3}$ are such that

$$
\begin{aligned}
& c_{1}(k+1, l+1)=\frac{\left(z_{k+1}^{l+1}{ }^{2}-1\right) z_{k}^{l}}{\left(z_{k+1}^{l}{ }^{2}-1\right) z_{k}^{l+1}} c_{1}(k+1, l), \\
& c_{1}(k, l+1)=\frac{\left(z_{k}^{l+1^{2}}-1\right) z_{k}^{l}}{\left(z_{k}^{l 2}-1\right) z_{k}^{l+1}} c_{1}(k, l), \\
& c_{1}(k+2, l)=\frac{\left(z_{k+2}^{l}{ }^{2}-1\right)}{\left(z_{k}^{l^{2}}-1\right)} c_{1}(k, l)
\end{aligned}
$$

and

$$
\begin{aligned}
& c_{3}(k+1, l-1)=\frac{\left(1-z_{k+1}^{l-1}\right) z_{k}^{l}}{\left(1-z_{k}^{l-1}\right) z_{k+1}^{l}} c_{3}(k, l-1), \\
& c_{3}(k, l+1)=\frac{\left(1-z_{k}^{l+1^{2}}\right)}{\left(1-z_{k}^{l-1^{2}}\right)} c_{3}(k, l-1), \\
& c_{3}(k+1, l)=\frac{\left(1-z_{k+1}^{l}{ }^{2}\right) z_{k}^{l}}{\left(1-z_{k}^{l^{2}}\right) z_{k+1}^{l}} c_{3}(k, l) .
\end{aligned}
$$

Here, $\left(F_{2}, G_{2}\right)$ is trivial.

\section{On multipliers/characteristics}

The relationship between the characteristics and conserved vectors of PDEs was well known for variational equations and was generalized relatively recently. In fact, the characteristics are the conserved vectors. The use of these in the construction of conservation laws has been discussed in detail in $[16,17]$ and [18] for the symmetry underlying relation that exists. Very recently, this idea has been initiated and discussed for PDEs in [19]. Below, we present the multipliers for the nontrivial cases of conservation laws that arise above. 


\subsection{The discrete Liouville equation}

For the discrete Liouville equation,

$$
u_{k}^{l} u_{k+1}^{l+1}=u_{k}^{l+1} u_{k+1}^{l}+z_{k}^{l}
$$

we have shown that

$$
\left(S_{k}-\mathrm{id}\right) F_{1}+\left(S_{l}-\mathrm{id}\right) G_{1}=\left(-\frac{1}{u_{k}^{l} u_{k+1}^{l}}+\frac{z_{k}^{l+1}}{z_{k}^{l} u_{k}^{l+1} u_{k+1}^{l+1}}\right)\left(-u_{k}^{l+1} u_{k+1}^{l}-z_{k}^{l}+u_{k}^{l} u_{k+1}^{l+1}\right) .
$$

Therefore, the multiplier is given by

$$
\lambda_{l 1}=-\frac{1}{u_{k}^{l} u_{k+1}^{l}}+\frac{z_{k}^{l+1}}{z_{k}^{l} u_{k}^{l+1} u_{k+1}^{l+1}}
$$

Similarly,

$$
\left(S_{k}-\mathrm{id}\right) F_{3}+\left(S_{l}-\mathrm{id}\right) G_{3}=\left(-\frac{1}{u_{k}^{l} u_{k}^{l+1}}+\frac{z_{k+1}^{l}}{z_{k}^{l} u_{k+1}^{l} u_{k+1}^{l+1}}\right)\left(-u_{k}^{l+1} u_{k+1}^{l}-z_{k}^{l}+u_{k}^{l} u_{k+1}^{l+1}\right)
$$

and the multiplier is given by

$$
\lambda_{l 3}=-\frac{1}{u_{k}^{l} u_{k}^{l+1}}+\frac{z_{k+1}^{l}}{z_{k}^{l} u_{k+1}^{l} u_{k+1}^{l+1}} \text {. }
$$

\subsection{The discrete sine-Gordon equation}

Consider the sine-Gordon equation

$$
u_{k+1}^{l+1} u_{k}^{l}=\frac{1+z_{k}^{l} u_{k}^{l+1} u_{k+1}^{l}}{u_{k}^{l+1} u_{k+1}^{l}+z_{k}^{l}}
$$

We have

$$
\begin{aligned}
\left(S_{k}-\mathrm{id}\right) F_{1}+\left(S_{l}-\mathrm{id}\right) G_{1}= & \left(u_{k}^{l+1} u_{k+1}^{l}+z_{k}^{l}\right)\left[\frac{c_{1}(k+1, l)}{\left(1-z_{k+1}^{l}\right) u_{k}^{l} u_{k}^{l+1}}-\frac{c_{1}}{\left(1-z_{k}^{l^{2}}\right) u_{k+1}^{l} u_{k+1}^{l+1}}\right] \\
& \times\left(-u_{k+1}^{l+1} u_{k}^{l}+\frac{1+z_{k}^{l} u_{k}^{l+1} u_{k+1}^{l}}{u_{k}^{l+1} u_{k+1}^{l}+z_{k}^{l}}\right)
\end{aligned}
$$

and

$$
\begin{aligned}
\left(S_{k}-\mathrm{id}\right) F_{3}+\left(S_{l}-\mathrm{id}\right) G_{3}= & -\left(u_{k}^{l+1} u_{k+1}^{l}+z_{k}^{l}\right)\left[\frac{c_{3}(k, l-1)}{\left.\left(1-z_{k}^{l-1}\right)^{2}\right) u_{k}^{l} u_{k+1}^{l}}-\frac{c_{3}}{\left(1-z_{k}^{l^{2}}\right) u_{k}^{l+1} u_{k+1}^{l+1}}\right] \\
& \times\left(-u_{k+1}^{l+1} u_{k}^{l}+\frac{1+z_{k}^{l} u_{k}^{l+1} u_{k+1}^{l}}{u_{k}^{l+1} u_{k+1}^{l}+z_{k}^{l}}\right) .
\end{aligned}
$$

The multipliers are then given by

$$
\lambda_{S g 1}=\left(u_{k}^{l+1} u_{k+1}^{l}+z_{k}^{l}\right)\left(\frac{c_{1}(k+1, l)}{\left(1-z_{k+1}^{l}{ }^{2}\right) u_{k}^{l} u_{k}^{l+1}}-\frac{c_{1}}{\left(1-z_{k}^{l^{2}}\right) u_{k+1}^{l} u_{k+1}^{l+1}}\right)
$$


and

$$
\lambda_{S g 2}=-\left(u_{k}^{l+1} u_{k+1}^{l}+z_{k}^{l}\right)\left(\frac{c_{3}(k, l-1)}{\left(1-z_{k}^{l-1^{2}}\right) u_{k}^{l} u_{k+1}^{l}}-\frac{c_{3}}{\left(1-z_{k}^{l^{2}}\right) u_{k}^{l+1} u_{k+1}^{l+1}}\right),
$$

respectively.

\section{Symmetries}

Again, we assume that the PDE is of the form (1), i.e.,

$$
u_{k+1}^{l+1}=\omega\left(k, l, u_{k}^{l}, u_{k+1}^{l}, u_{k}^{l+1}\right)
$$

Consider the transformation [1]

$$
\Gamma:\left(k, l, u_{k}^{l}, u_{k+1}^{l}, u_{k}^{l+1}, u_{k+1}^{l+1}\right) \mapsto\left(k, l, \hat{u}_{k}^{l}, \hat{u}_{k+1}^{l}, \hat{u}_{k}^{l+1}, \hat{u}_{k+1}^{l+1}\right) .
$$

We know that a symmetry is a one-parameter group of transformations that maps solutions into solutions. If we assume that $\Gamma$ is a symmetry for (61) then we have

$$
\hat{u}_{k+1}^{l+1}=\omega\left(k, l, \hat{u}_{k}^{l}, \hat{u}_{k+1}^{l}, \hat{u}_{k}^{l+1}\right)
$$

whenever (61) holds. Lie symmetries are obtained by linearizing the symmetry condition about the identity, as follows. We seek one-parameter (local) Lie groups of symmetries of the form

$$
\hat{u}_{k}^{l}=u_{k}^{l}+\epsilon Q\left(k, l, u_{k}^{l}\right)+O\left(\epsilon^{2}\right) .
$$

The function $Q$ is called the characteristic of the one-parameter group. We also have

$$
\begin{aligned}
& \hat{u}_{k+1}^{l}=u_{k+1}^{l}+\epsilon Q\left(k+1, l, u_{k+1}^{l}\right)+O\left(\epsilon^{2}\right), \\
& \hat{u}_{k}^{l+1}=u_{k}^{l+1}+\epsilon Q\left(k, l+1, u_{k}^{l+1}\right)+O\left(\epsilon^{2}\right), \\
& \hat{u}_{k+1}^{l+1}=u_{k+1}^{l+1}+\epsilon Q\left(k+1, l+1, u_{k+1}^{l+1}\right)+O\left(\epsilon^{2}\right) .
\end{aligned}
$$

Expanding (64)-(67) to first order in $\epsilon$ yields the linearized symmetry condition

$$
S_{k} S_{l} Q-X \omega=0
$$

where

$$
X=Q \frac{\partial}{\partial u_{k}^{l}}+\left(S_{k} Q\right) \frac{\partial}{\partial u_{k+1}^{l}}+\left(S_{l} Q\right) \frac{\partial}{\partial u_{k}^{l+1}}
$$

\subsection{The discrete Liouville equation}

Consider the discrete Liouville equation [8]

$$
u_{k}^{l} u_{k+1}^{l+1}=u_{k}^{l+1} u_{k+1}^{l}+z_{k}^{l}
$$


This equation can be written in the form

$$
u_{k+1}^{l+1}=\omega\left(k, l, u_{k}^{l}, u_{k}^{l+1}, u_{k+1}^{l}\right),
$$

where $\omega=\left(u_{k}^{l+1} u_{k+1}^{l}+z_{k}^{l}\right) / u_{k}^{l}$. We aim to find one-parameter Lie groups of symmetries by assuming that the characteristic is of the form

$$
Q=Q\left(k, l, u_{k}^{l}\right)
$$

A linearized symmetry condition for (71) is given by

$$
\begin{aligned}
& \left(S_{k} S_{l}\right) Q\left(k, l, u_{k}^{l}\right)-X \omega=0, \\
& Q(k+1, l+1, \omega)-X \omega=0,
\end{aligned}
$$

where $X$ is the symmetry generator given by

$$
X=Q\left(k, l, u_{k}^{l}\right) \frac{\partial}{\partial u_{k}^{l}}+Q\left(k+1, l, u_{k+1}^{l}\right) \frac{\partial}{\partial u_{k+1}^{l}}+Q\left(k, l+1, u_{k}^{l+1}\right) \frac{\partial}{\partial u_{k}^{l+1}} .
$$

The symmetry condition (73) becomes

$$
\begin{aligned}
& Q(k+1, l+1, \omega)+\left(\frac{u_{k}^{l+1} u_{k+1}^{l}+z_{k}^{l}}{u_{k}^{l 2}}\right) Q-\left(\frac{u_{k}^{l+1}}{u_{k}^{l}}\right) Q\left(k+1, l, u_{k+1}^{l}\right) \\
& -\left(\frac{u_{k+1}^{l}}{u_{k}^{l}}\right) Q\left(k, l+1, u_{k}^{l+1}\right)=0 .
\end{aligned}
$$

We differentiate (74) with respect to $u_{k}^{l}$ (keeping $\omega$ fixed), i.e., we apply the differential operator $L_{0}=\left(\partial / \partial u_{k}^{l}\right)+\left(\partial u_{k+1}^{l} / \partial u_{k}^{l}\right)\left(\partial / \partial u_{k+1}^{l}\right)$. This gives

$$
\begin{gathered}
\left(\frac{\partial}{\partial u_{k}^{l}}+\frac{u_{k}^{l+1} u_{k+1}^{l}+z_{k}^{l}}{u_{k}^{l} u_{k}^{l+1}} \frac{\partial}{\partial u_{k+1}^{l}}\right)\left[Q(k+1, l+1, w)+\left(\frac{u_{k}^{l+1} u_{k+1}^{l}+z_{k}^{l}}{u_{k}^{l^{2}}}\right) Q\right. \\
\left.-\left(\frac{u_{k}^{l+1}}{u_{k}^{l}}\right) Q\left(k+1, l, u_{k+1}^{l}\right)-\left(\frac{u_{k+1}^{l}}{u_{k}^{l}}\right) Q\left(k, l+1, u_{k}^{l+1}\right)\right]=0,
\end{gathered}
$$

i.e.,

$$
\begin{gathered}
\left(\frac{Q^{\prime} u_{k}^{l^{2}}-2 u_{k}^{l} Q}{u_{k}^{l^{2}}}\right)\left[\left(u_{k}^{l+1} u_{k+1}^{l}+z_{k}^{l}\right)+\left(S_{k} Q\right) u_{k}^{l+1}+\left(S_{l} Q\right) u_{k+1}^{l}\right] \\
+\left(\frac{u_{k}^{l+1} u_{k+1}^{l}+z_{k}^{l}}{u_{k}^{l}}\right)\left[Q \frac{u_{k}^{l+1}}{u_{k}^{l}}-\left(S_{k} Q\right)^{\prime} u_{k}^{l+1}-\left(S_{l} Q\right)\right]=0 .
\end{gathered}
$$

We now differentiate (76) with respect to $u_{k}^{l}$ to get

$$
\left(u_{k}^{l+1} u_{k+1}^{l}+z_{k}^{l}\right)\left[\frac{Q}{u_{k}^{l}}\right]^{\prime}+\left(u_{k}^{l+1} u_{k+1}^{l}+z_{k}^{l}\right)\left[\frac{Q^{\prime} u_{k}^{l^{2}}-2 u_{k}^{l} Q}{u_{k}^{l^{2}}}\right]^{\prime}=0 \text {. }
$$


This simplifies to

$$
\left[\frac{Q\left(k, l, u_{k}^{l}\right)}{u_{k}^{l}}\right]^{\prime}=-\left[\frac{Q^{\prime}\left(k, l, u_{k}^{l}\right) u_{k}^{l}-2 Q\left(k, l, u_{k}^{l}\right)}{u_{k}^{l}}\right]^{\prime} .
$$

Integrating (78) with respect to $u_{k}^{l}$, and rearranging the resulting equation leads to

$$
Q^{\prime}\left(k, l, u_{k}^{l}\right)-\frac{1}{u_{k}^{l}} Q\left(k, l, u_{k}^{l}\right)-c_{1}=0 .
$$

The general solution to (79) is given by

$$
Q\left(k, l, u_{k}^{l}\right)=c_{1}(k, l) u_{k}^{l} \ln \left(u_{k}^{l}\right)+c_{2}(k, l) u_{k}^{l}
$$

where $c_{1}$ and $c_{2}$ are functions of $k$ and $l$. We then substitute (80) in (74) to get the following constraint:

$$
\begin{aligned}
& {\left[c_{2}(k+1, l+1)+c_{2}(k, l)-c_{2}(k+1, l)-c_{2}(k, l+1)\right] u_{k}^{l+1} u_{k+1}^{l}} \\
& \quad+\left[c_{2}(k+1, l+1)+c_{2}(k, l)\right] z_{k}^{l}=0
\end{aligned}
$$

The function $c_{2}$ does not depend on $u_{k}^{l+1}$ and $u_{k+1}^{l}$; therefore we can say that

$$
\begin{aligned}
& c_{1}=0, \\
& c_{2}(k+1, l+1)=-c_{2}(k, l), \quad c_{2}(k+1, l)=-c_{2}(k, l+1) .
\end{aligned}
$$

It can easily be verified that the functions that satisfy (82) are given by

$$
c_{2}(k, l)=(-1)^{k}, \quad c_{2}(k, l)=(-1)^{l} .
$$

Therefore, the symmetries of the Liouville equation (6) are as follows:

$$
\begin{aligned}
& X_{1}=(-1)^{k} u_{k}^{l} \frac{\partial}{\partial u_{k}^{l}}-(-1)^{k} u_{k+1}^{l} \frac{\partial}{\partial u_{k+1}^{l}}+(-1)^{k} u_{k}^{l+1} \frac{\partial}{\partial u_{k}^{l+1}} \\
& X_{2}=(-1)^{l}\left(u_{k}^{l} \frac{\partial}{\partial u_{k}^{l}}+u_{k+1}^{l} \frac{\partial}{\partial u_{k+1}^{l}}-u_{k}^{l+1} \frac{\partial}{\partial u_{k}^{l+1}}\right) .
\end{aligned}
$$

\subsection{The discrete sine-Gordon equation}

The discrete sine-Gordon equation is given by [8]

$$
u_{k+1}^{l+1} u_{k}^{l}=\frac{1+z_{k}^{l} u_{k}^{l+1} u_{k+1}^{l}}{u_{k}^{l+1} u_{k+1}^{l}+z_{k}^{l}}
$$

Imposing the symmetry condition (68) on the discrete sine-Gordon equation, we obtain

$$
\begin{aligned}
& Q\left(k+1, l+1, u_{k+1}^{l+1}\right)+\left[\frac{z_{k}^{l} u_{k}^{l+1} u_{k+1}^{l}+1}{u_{k}^{l^{2}}\left(z_{k}^{l}+u_{k}^{l+1} u_{k+1}^{l}\right)}\right] Q-\frac{Q\left(k+1, l, u_{k+1}^{l}\right)}{u_{k}^{l}}\left[\frac{z_{k}^{l 2} u_{k}^{l+1}-u_{k}^{l+1}}{\left(u_{k+1}^{l} u_{k}^{l+1}+z_{k}^{l}\right)^{2}}\right] \\
& -\left(\frac{Q\left(k, l+1, u_{k}^{l+1}\right)}{u_{k}^{l}}\right)\left[\frac{z_{k}^{l^{2}} u_{k+1}^{l}-u_{k+1}^{l}}{\left(u_{k+1}^{l} u_{k}^{l+1}+z_{k}^{l}\right)^{2}}\right]=0 .
\end{aligned}
$$


The function in above equation is associated with different arguments. To overcome this, we differentiate the above equation with to $u_{k}^{l}$. This yields

$$
\begin{gathered}
\frac{\left(z_{k}^{l^{2}}-1\right)\left[u_{k}^{l+1} Q\left(k+1, l, u_{k+1}^{l}\right)+u_{k+1}^{l} Q\left(k, l+1, u_{k}^{l+1}\right)\right]}{\left(u_{k+1}^{l} u_{k}^{l+1}+z_{k}^{l}\right)^{2} u_{k}^{l^{2}}}+\frac{\left(z_{k}^{l} u_{k+1}^{l} u_{k}^{l+1}+1\right)}{\left(u_{k+1}^{l} u_{k}^{l+1}+z_{k}^{l}\right)} \\
\times \frac{d}{d u_{k}^{l}}\left(\frac{Q}{u_{k}^{l^{2}}}\right)+\frac{\left(z_{k}^{l} u_{k+1}^{l} u_{k}^{l+1}+1\right) Q}{u_{k}^{l^{3}}\left(u_{k}^{l+1} u_{k+1}^{l}+z_{k}^{l}\right)}-\frac{\left(z_{k}^{l} u_{k+1}^{l} u_{k}^{l+1}+1\right)\left(u_{k+1}^{l} u_{k}^{l+1}+z_{k}^{l}\right)}{u_{k}^{l^{2}}} \\
\times\left[\frac{d}{d u_{k+1}^{l}}\left(\frac{Q\left(k+1, l, u_{k+1}^{l}\right)}{\left(u_{k+1}^{l} u_{k}^{l+1}+z_{k}^{l}\right)^{2}}\right)-\frac{1}{u_{k}^{l+1}} \frac{d}{d u_{k+1}^{l}}\left(\frac{u_{k+1}^{l}}{\left(u_{k+1}^{l} u_{k}^{l+1}+z_{k}^{l}\right)^{2}}\right)\right]=0 .
\end{gathered}
$$

We can solve for $Q$ after a series of steps. Firstly, we multiply (88) by $\left(u_{k}^{l}\right)^{2}$. Secondly, we differentiate the resulting equation with respect to $u_{k}^{l}$. Finally, we simplify to get the equation

$$
\frac{d}{d u_{k}^{l}}\left(\frac{Q^{\prime}\left(k, l, u_{k}^{l}\right) u_{k}^{l}-Q\left(k, l, u_{k}^{l}\right)}{u_{k}^{l}}\right)=0 .
$$

The general solution of (89) is given by

$$
Q\left(k, l, u_{k}^{l}\right)=B(k, l) u_{k}^{l} \ln u_{k}^{l}+C(k, l) u_{k}^{l},
$$

where $B$ and $C$ are functions of $k$ and $l$. The substitution of (90) in (89) and (87) puts more constraints on the unknown functions $B$ and $C$. After a set of long calculations we get

$$
Q\left(k, l, u_{k}^{l}\right)=(-1)^{k} u_{k}^{l}, \quad Q\left(k, l, u_{k}^{l}\right)=(-1)^{l} u_{k}^{l} .
$$

Therefore, the symmetries are given by

$$
\begin{aligned}
& X_{1}=(-1)^{k}\left(u_{k}^{l} \frac{\partial}{\partial u_{k}^{l}}-u_{k+1}^{l} \frac{\partial}{\partial u_{k+1}^{l}}+u_{k}^{l+1} \frac{\partial}{\partial u_{k}^{l+1}}\right), \\
& X_{2}=(-1)^{l}\left(u_{k}^{l} \frac{\partial}{\partial u_{k}^{l}}+u_{k+1}^{l} \frac{\partial}{\partial u_{k+1}^{l}}-u_{k}^{l+1} \frac{\partial}{\partial u_{k}^{l+1}}\right) .
\end{aligned}
$$

\section{Concluding remarks}

In this paper, we have obtained conservation laws, multipliers, and symmetries of the discrete Liouville equation and the discrete sine-Gordon equation. It should be noted that when we were finding the conservation laws, the differentiations have created a hierarchy of functional difference equations that $F$ and $G$ satisfied. The unknown functions were naturally found by going up the hierarchy but surprisingly we were also able to find another constraint on $z$. In fact the shift operator, $S_{l}$, acts on (50) to produce the following equation:

$$
S_{l}\left(c_{3}(k+1, l-1)\right)=c_{3}(k+1, l)=\frac{\left(1-z_{k+1}^{l}{ }^{2}\right) z_{k}^{l+1}}{\left(1-z_{k}^{l^{2}}\right) z_{k+1}^{l+1}} c_{3}(k, l) .
$$

By replacing in (94) the function $c_{3}(k+1, l)$ with its expression given by (52), we obtained the constraint (7), i.e., $z_{k+1}^{l+1} z_{k}^{l}-z_{k}^{l+1} z_{k+1}^{l}=0$. The constraint (7) turns out to be a sufficient 
condition for the discrete Liouville equation and the discrete sine-Gordon equation to have nontrivial conservation laws (note that the substitution of (35), (36), and (37) into (8) leads to the same constraint (7)). This condition was obtained in [20] using the singularity confinement condition. It is also precisely the one obtained in [8] using the techniques of the study of the degree of the iterate.

We note that the association of symmetries, conservation laws, and integrability for difference equations is as important and conclusive as was established for differential equations even in the non-variational case.

\section{Competing interests}

The authors declare that they have no competing interests.

\section{Authors' contributions}

All authors read and approved the final manuscript.

\section{Acknowledgements}

MKF-G and AHK would like to thank the referees of this manuscript for their valuable comments and suggestions.

Received: 10 March 2014 Accepted: 29 July 2014 Published: 15 Aug 2014

\section{References}

1. Hydon, P: Symmetries and first integrals of ordinary difference equations. Proc. R. Soc. Lond. A 456, 2835-2855 (2000)

2. Levi, D, Vinet, L, Winternitz, P: Lie group formalism for difference equations. J. Phys. A, Math. Gen. 30, 633-649 (1997)

3. Levi, D, Winternitz, P: Symmetries of discrete dynamical systems. J. Math. Phys. 37, 5551-5576 (1996)

4. Quispel, GRW, Sahadevan, R: Lie symmetries and integration of difference equations. Phys. Lett. A 184, 64-70 (1993)

5. Hydon, PE: Conservation laws of partial difference equations with two independent variables. J. Phys. A, Math. Gen. 34, 10347-10355 (2001)

6. Rasin, OG, Hydon, PE: Conservation laws of discrete Korteweg-de Vries equation. SIGMA 1, 1-6 (2005)

7. Hydon, P, Mansfield, E: A variational complex for difference equations. Found. Comput. Math. 4, 187-217 (2004)

8. Tremblay, S, Grammaticos, B, Ramani, A: Integrable lattice equations and their growth properties. Phys. Lett. A 278, 319-324 (2001)

9. Hirota, R: Nonlinear partial difference equations. III. Discrete sine-Gordon equation. J. Phys. Soc. Jpn. 43, 2079-2086 (1977)

10. Bobenko, A, Bordemann, M, Gunn, C, Pinkall, U: On two integrable cellular automata. Commun. Math. Phys. 158, 127-134 (1993)

11. Kara, AH, Mahomed, FM: Relationship between symmetries and conservation laws. Int. J. Theor. Phys. 39(1), 23-40 (2000)

12. Agarwal, RP, El-Sayed, AMA, Salman, SM: Fractional-order Chuas system: discretization, bifurcation and chaos. Adv. Differ. Equ. 2013, 320 (2013)

13. Wu, GC, Baleanu, D: Discrete fractional logistic map and its chaos. Nonlinear Dyn. 75, 283-287 (2014)

14. Wu, GC, Baleanu, D: Discrete chaos in fractional delayed logistic maps. Nonlinear Dyn. (2014). doi:10.1007/s11071-014-1250-3

15. Wu, GC, Baleanu, D: Chaos synchronization of the discrete fractional logistic map. Signal Process. 102, 96-99 (2014)

16. Anco, SC, Bluman, G: Direct construction method for conservation laws of partial differential equations. I. Examples of conservation law classifications. Eur. J. Appl. Math. 13, 545-566 (2002)

17. Anco, SC, Bluman, G: Direct construction method for conservation laws of partial differential equations. II. General treatment. Eur. J. Appl. Math. 13, 567-585 (2002)

18. Kara, AH: A symmetry invariance analysis of the multipliers \& conservation laws of the Jaulent-Miodek and some families of systems of KdV type equations. J. Nonlinear Math. Phys. 16, suppl., 149-156 (2009)

19. Grant, TJ, Hydon, PE: Characteristics of conservation laws for difference equations. Found. Comput. Math. 13, 667-692 (2013)

20. Papageorgiou, V, Grammaticos, B, Ramani, A: Integrable lattices and convergence acceleration algorithms. Phys. Lett. A 179, 111-115 (1993)

10.1186/1687-1847-2014-224

Cite this article as: Folly-Gbetoula and Kara: Symmetries, conservation laws, and 'integrability' of difference

equations. Advances in Difference Equations 2014, 2014:224 